\title{
Network Allocation Vector-based dynamic backoff algorithm for IEEE 802.11 DCF
}

\author{
In-Soo Lee ${ }^{1}$, Dong-Won Kum ${ }^{2 \mathrm{a})}$, Won-Kyeong Seo ${ }^{2 \mathrm{~b})}$, \\ and You-Ze Cho ${ }^{2 \mathrm{c})}$ \\ ${ }^{1}$ Engineering Business Center, KT, Gyeonggi-do, Korea \\ ${ }^{2}$ School of Electrical Engineering and Computer Science, Kyungpook National \\ University, Daegu, Korea \\ In-Soo Lee and Dong-Won Kum contributed equally to this work \\ You-Ze Cho is a corresponding author \\ a)80kumsy@ee.knu.ac.kr \\ b)morglory@ee.knu.ac.kr \\ c) yzcho@ee.knu.ac.kr
}

\begin{abstract}
This paper presents a novel backoff algorithm based on a Network Allocation Vector (NAV) for the IEEE 802.11 Distributed Coordination Function (DCF). In the DCF, the NAV indicates the amount of time that a medium will be reserved by other nodes, so it provides an accurate estimation of neighbor nodes activities around a node. Thus, the proposed scheme dynamically adjusts the contention window according to the variation of neighbor nodes activities, which is estimated by NAV measurements. Simulation results demonstrate that the proposed scheme outperforms existing schemes in terms of total throughput and fairness between nodes.
\end{abstract}

Keywords: backoff algorithm, dynamic contention window, Network Allocation Vector (NAV), IEEE 802.11 DCF

Classification: Science and engineering for electronics

\section{References}

[1] IEEE Standard for Wireless LAN Medium Access Control (MAC) and Physical Layer (PHY) Specifications, IEEE Std 802.11, 2007.

[2] N. Song, B. Kwak, J. Song, and L. E. Miller, "Enhancement of IEEE 802.11 distributed coordination function with exponential increase exponential decrease backoff algorithm," Proc. IEEE VTC 2003-Spring, April 2003.

[3] V. Bharghavan, A. Demers, S. Shenker, and L. Zhang, "MACAW: a media access protocol for wireless LAN's," Proc. ACM SIGCOMM, Sept. 1994.

[4] J. Deng, P. K. Varshney, and Z. J. Haas, "A new backoff algorithm for the IEEE 802.11 distributed coordination function," Proc. CNDS, Jan. 2004.

[5] G. Bianchi, "Performance analysis of the IEEE 802.11 distributed coordination function," IEEE J. Sel. Areas Commun., vol. 18, pp. 535-547, March 2000. 
[6] H.-C. Lin, Y.-C. Chang, I.-C. Liu, and S.-T. Liang, "Study on the enhanced back-off schemes for the IEEE 802.11 wireless LANs," Proc. APWCS, Aug. 2007.

[7] R. Jain, The Art of Computer Systems Performance Analysis: Techniques for Experimental Design, Measurement, Simulation and Modeling, Wiley, 1991.

\section{Introduction}

The IEEE 802.11 Medium Access Control (MAC) standard consists of two coordination functions: the Distributed Coordination Function (DCF) and Point Coordination Function (PCF), where the DCF is the fundamental access mechanism, while the PCF is used optionally. In the DCF, active nodes compete for channel access in a distributed manner using the Carrier Sense Multiple Access with Collision Avoidance (CSMA/CA) scheme [1].

In the CSMA/CA, when a channel is sensed as busy, each competing node backoff its frame transmission at a randomly chosen backoff time to reduce the possibility of collision. This backoff time is selected uniformly between 0 and the Contention Window (CW). Meanwhile, in the IEEE 802.11 DCF, the CW is dynamically controlled by a backoff algorithm, called the Binary Exponential Backoff (BEB). In the BEB algorithm, the CW is doubled every time a node experiences a frame collision. If a node is successful in its data frame transmission, the $\mathrm{CW}$ is reset to the minimum value. To avoid the $\mathrm{CW}$ from growing too large or becoming too small, two boundaries are defined: the maximum contention window $\left(\mathrm{CW}_{\max }\right)$ and minimum contention window $\left(\mathrm{CW}_{\min }\right)[1]$.

However, when increasing the number of active nodes in a network, BEB results in a serious reduction of network throughput, as it does not use the collision history of the previous frames and reduces the CW too quickly, making it unsuitable for a network under a heavy load [2]. Furthermore, the BEB scheme suffers from a fairness problem as some nodes can achieve a significantly higher throughput than others. This fairness problem occurs due to the fact that $\mathrm{BEB}$ resets the $\mathrm{CW}$ of a successful sender to $\mathrm{CW}_{\min }$, while other nodes continue to maintain larger contention windows [3, 4].

To enhance the performance of BEB, various backoff algorithms have already been proposed, such as Exponential Increase and Exponential Decrease (EIED) [2], Multiplicative Increase and Linear Decrease (MILD) [3], and Linear/Multiplicative Increase and Linear Decrease (LMILD) [4]. Table I compares the CW setting methods of these algorithms. All of these algorithms use the events of successful transmission or collision to control the CW. Plus, LMILD exploits events of overhearing successful transmission or collision to control the CW.

In [5], the author derived the optimum CW to maximize the network throughput for a fully connected network with exactly $N$ active nodes. He showed that the optimum $\mathrm{CW}$ is proportional to the number $N$ of active 
Table I. Comparison of $\mathrm{CW}$ setting methods in BEB, EIED, MILD, and LMILD.

\begin{tabular}{|c|c|c|}
\hline $\begin{array}{l}\text { Events } \\
\text { Schemes }\end{array}$ & $\begin{array}{l}\text { (1) Upon collision } \\
\text { (3) Overhearing collision }\end{array}$ & $\begin{array}{l}\text { (2) Upon successful transmission } \\
\text { (4) Overhearing successful transmission }\end{array}$ \\
\hline BEB & $(1) \mathrm{CW}=\min \left(\mathrm{CW}^{*} 2, \mathrm{CW}_{\max }\right)$ & (2) $\mathrm{CW}=\mathrm{CW}_{\min }$ \\
\hline EIED & (1) $\mathrm{CW}=\min \left(\mathrm{CW}^{*} \gamma_{\mathrm{I}}, \mathrm{CW}_{\max }\right)$ & (2) $\mathrm{CW}=\max \left(\mathrm{CW} / \gamma_{\mathrm{D}}, \mathrm{CW}_{\min }\right)$ \\
\hline MILD & (1) $\mathrm{CW}=\min \left(\mathrm{CW}^{*} 1.5, \mathrm{CW}_{\max }\right)$ & (2) $\mathrm{CW}=\max \left(\mathrm{CW}-1, \mathrm{CW}_{\min }\right)$ \\
\hline LMILD & $\begin{array}{l}\text { (1) } \mathrm{CW}=\min \left(\mathrm{CW}^{*} \mathrm{~m}_{\mathrm{c}}, \mathrm{CW}_{\max }\right) \\
\text { (3) } \mathrm{CW}=\min \left(\mathrm{CW}+\mathrm{l}_{\mathrm{c}}, \mathrm{CW}_{\max }\right)\end{array}$ & $(2) /(4) \mathrm{CW}=\max \left(\mathrm{CW}-\mathrm{l}_{\mathrm{s}}, \mathrm{CW}_{\min }\right)$ \\
\hline \multicolumn{3}{|c|}{$\begin{array}{l}\text { - The values of } \gamma_{I} \text { and } \gamma_{D} \text { are the factor for increasing and decreasing the } \mathrm{CW} \text {, respectively. } \\
\text { - The values of } \mathrm{m}_{\mathrm{c}} \text { and } 1_{\mathrm{c}} \text { are the factor for increasing the } \mathrm{CW} \text {. } \\
\text { - The value of } 1_{\mathrm{s}} \text { is the factor for decreasing the } \mathrm{CW} \text {. }\end{array}$} \\
\hline
\end{tabular}

nodes in the network. Therefore, a well-designed backoff algorithm should adjust the $\mathrm{CW}$ depending on the actual nodes activities. Yet, all of the above mentioned algorithms exploit only binary information about successful transmission or collision to control the CW.

In the IEEE 802.11 DCF, the Network Allocation Vector (NAV) indicates the amount of time that a medium will be reserved by other nodes. Thus, the NAV is a good indicator for the busyness of the medium, so it provides an accurate estimation of neighbor nodes activities around a node.

In this paper, we propose a novel NAV-based Backoff Algorithm (NABA) for the IEEE 802.11 DCF to improve the network throughput and fairness between nodes. The proposed NABA adjusts the CW dynamically according to the variation of neighbor nodes activities, which is estimated by NAV measurements.

\section{NABA: NAV-based Backoff Algorithm}

The IEEE 802.11 DCF uses NAV for the virtual carrier sensing [1]. Whenever a node overhears Request-to-Send (RTS), Clear-to-Send (CTS) or data frames, it sets its NAV to the value obtained from the duration field in the MAC frames. The NAV is then only updated when the new NAV value is greater than the current NAV value.

In NABA, a node uses NAV measurements to estimate the neighbor nodes activities. Each node computes its own $N A V_{-} S U M$, which is the sum of the non-overlapped NAV values during a measurement interval $\tau$. In order to mitigate the effect of traffic bursts, an exponentially weighted moving average of $N A V_{-} S U M$ is used to estimate the average busy portion as follows.

$$
\overline{N A V_{-} S U M_{j}}=\alpha \cdot \overline{N A V_{-} S U M_{j-1}}+(1-\alpha) \cdot N A V_{-} S U M_{j},
$$

where $\alpha$ is a weight factor in the range of $[0,1]$ and $j$ refers to the $j$ th update period. Since $\overline{N A V_{-} S U M_{j}}$ indicates the average busy portion by neighbor nodes on the shared medium during the measurement interval $\tau$, $\overline{N A V_{-} S U M_{j}}$ over $\tau$ provides the neighbor nodes activities for the $j$ th update period. Therefore, we can estimate the variation ratio $\gamma$ of neighbor nodes activities as

$$
\gamma=\frac{\overline{N A V_{-} S U M_{j}}}{\overline{N A V_{-} S U M_{j-1}}}
$$


Every measurement interval $\tau$, NABA adjusts the CW dynamically according to the variation ratio $\gamma$ of neighbor nodes activities as follows. If $\gamma$ is greater than one, it increases the $\mathrm{CW}$ as

$$
C W=\min \left(C W \times \gamma, C W_{\max }\right) .
$$

If $\gamma$ is less than one, it decreases the $\mathrm{CW}$ as

$$
C W=\max \left(C W \times \gamma, C W_{\min }\right) .
$$

In addition to NABA, a source node can adjust its $\mathrm{CW}$ according to the events of successful transmission or collision. Thus, NABA can be combined with existing backoff schemes, such as BEB, EIED, and MILD.

To introduce NABA in the IEEE $802.11 \mathrm{DCF}$, it requires that a node should overhear frame transmissions by other nodes. Most of commercial WiFi LAN cards support active mode by default and some products do not support power management. However, if a node is in power save mode, it can use a conventional backoff algorithm, while other nodes in active mode perform backoff according to NABA.

\section{Performance evaluation}

In this section, we evaluate the performance of NABA in terms of collision ratio, throughput, and fairness through simulation using the NS-2. To evaluate the efficiency of NABA, we compared the performance of MILD+NABA with those of the BEB, EIED, and MILD backoff algorithms. The reason we chose MILD+NABA for the comparison was that MILD showed a better performance than the other schemes when NABA was combined with the BEB or EIED scheme. In [6], the authors showed that EIED outperformed BEB, MILD, and LMILD in terms of throughput. Therefore, we compared the performance of MILD+NABA with those of BEB, EIED, and MILD.

\subsection{Simulation environments}

In our simulation, we assumed that all the wireless nodes were stationary and within transmission range of each other. Each wireless node sent its data to the same corresponding wired node, which was connected to an access point. Also, we assumed that the IEEE 802.11 DCF used the RTS/CTS handshaking for all data transmissions. As traffic sources, we considered Constant Bit-Rate (CBR) flows and Variable Bit-Rate (VBR) flows. The VBR flows were generated by the Poisson process. As the transport protocol, we used the User Datagram Protocol (UDP) with a data packet size of 512 bytes. In the simulation, the $\alpha$ and $\tau$ parameter values were set at 0.1 and 1 second, respectively.

\subsection{Performance metrics}

For the performance comparison, we used the following metrics.

- Collision ratio: This was defined as the number of collisions divided by the number of data transmissions. 
- Total throughput: This was defined as the amount of data transmitted successfully through the network per unit time.

- Fairness index: This was defined as $\Psi=\left(\sum_{i=1}^{n} x_{i}\right)^{2} /\left(n \sum_{i=1}^{n} x_{i}^{2}\right)$, where $x_{i}$ is the throughput of a given flow $i[7]$.

\subsection{Simulation results}

In the simulation, VBR traffic flows were used to evaluate the collision ratio and total throughput, while CBR traffic flows with the same rate were used for the fairness index. The simulation was run for 300 seconds and each simulation experiment was repeated 20 times. In the simulation results, each curve indicates the average value with a $90 \%$ confidence interval.

Fig. 1 compares the collision ratio between MILD+NABA, BEB, EIED, and MILD when increasing the number of source nodes. Under heavy load (at 70 sources), MILD+NABA reduced the collision ratio by about $60 \%$ when compared with BEB and by about $12 \%$ when compared with EIED and MILD. The reason was that NABA could control the CW more optimally than the other schemes by considering the neighbor nodes activities.

Fig. 2 compares the total throughput between MILD+NABA, BEB, EIED, and MILD when increasing the number of source nodes. The collision ratio directly affects the total throughput. This figure shows that at a lower traffic

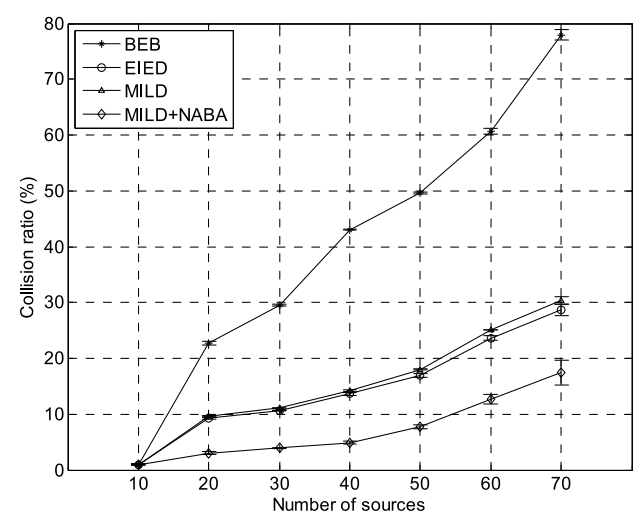

Fig. 1. Comparison of collision ratio.

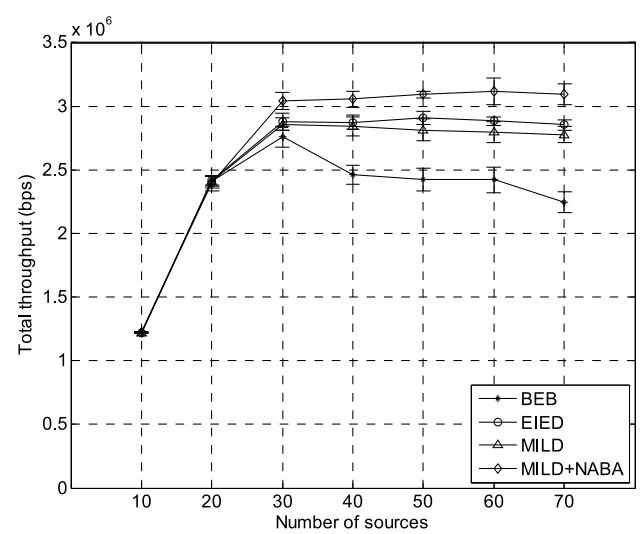

Fig. 2. Comparison of total throughput. 


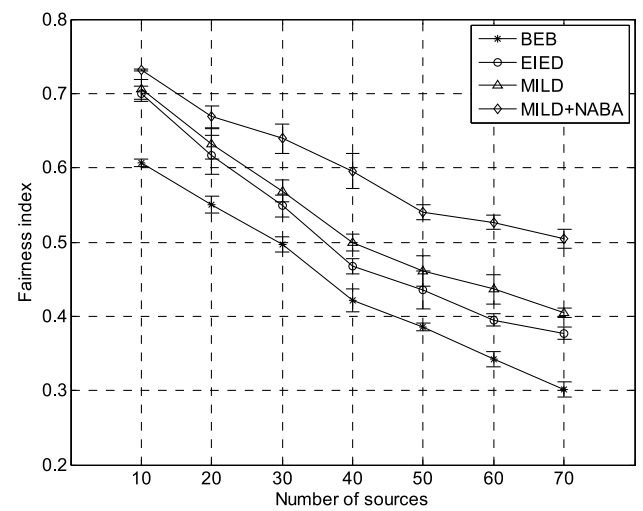

Fig. 3. Comparison of fairness index.

load (small number of source nodes), the total throughputs were similar for all the schemes. However, under heavy load (at 70 sources), due to the reduction of the bandwidth wasted by collisions and the amount of idle medium time, MILD+NABA improved the total throughput by more than $45 \%$ when compared with the BEB and by more than $14 \%$ when compared with the EIED and MILD schemes.

Fig. 3 compares the fairness index between MILD+NABA, BEB, EIED, and MILD when increasing the number of source nodes. The fairness index is a real value between 0 and 1 , where the values closer to 1 indicate a better fairness between nodes. This figure shows that MILD+NABA exhibited a better fairness than the other three backoff schemes. When the number of source nodes was 70, MILD+NABA improved the fairness index by more than $60 \%$ when compared with the BEB and by about $30 \%$ when compared with EIED and MILD schemes. The reason was that NABA had similar CW values among competing nodes, as every node always maintains its CW dynamically based on NAV measurements.

\section{Conclusion}

This paper proposed a novel NAV-based backoff algorithm, called NABA, for the IEEE 802.11 DCF to improve the network throughput and fairness between nodes. NABA dynamically adjusts the contention window according to the variation of neighbor nodes activities, which is estimated by NAV measurements. NABA can also be combined with existing backoff schemes, such as BEB, EIED, and MILD. Simulation results demonstrated that MILD+NABA could enhance the performance significantly in terms of the collision ratio, total throughput, and fairness when compared with BEB, EIED, and MILD.

\section{Acknowledgments}

This research was supported by the NRF (National Research Foundation of Korea) (2009-0076947), and the MKE (The Ministry of Knowledge Economy), Korea, under the ITRC (Information Technology Research Center) support program supervised by the NIPA (National IT Industry Promo- 
tion Agency) (NIPA-2010-(C1090-1011-0013)), and the IT R\&D program of MKE/KEIT (KI001822).

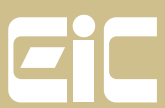

() IEICE 2010

DOI: 10.1587/elex.7.1571

Received August 24, 2010

Accepted September 15, 2010

Published October 25, 2010 